\title{
Identification and Control of Nonlinear Harmonic Coupling for Pulsed Jet Injection
}

\author{
Cory Hendrickson and Robert M'Closkey, Member, IEEE,
}

\begin{abstract}
This paper describes an approach to periodic reference tracking in a fundamental pulsed jet injection experimental study. The objective is to match the jet's temporal velocity profile to a periodic reference for purposes of studying the mixing dynamics between the jet and surrounding fluid. The challenge lies in controlling the highly nonlinear and poorly understood dynamics associated with the jet velocity. Although the actuator maintains good authority over the jet velocity, the nonlinear jet dynamics creates a high degree of coupling among neighboring harmonics that depends on the forcing level. We approach the problem by demodulating the jet velocity measurement at harmonic frequencies represented in the desired waveform into baseband components. In a neighborhood of a desired operating point, an empirical relationship is developed by perturbing the baseband components and measuring their effect on neighboring harmonics. We demonstrate that this relationship can be reasonably modeled as a linear MIMO gain for nearby operating points. This knowledge is exploited to create a stabilizing feedback control that asymptotically drives the jet velocity to its reference.
\end{abstract}

\section{INTRODUCTION}

The control of a jet injected into quiescent surroundings or into a crossflow is a fundamental problem with application to a wide range of engineering systems, particularly those for propulsion and energy generation [1], [2]. In turbine engines, for example, active control of the jet in crossflow has been shown to improve the spread and penetration of the jet into the crossflow for dilution jet injection [3] and improve boundary layer attachment at low turbine inlet Reynolds numbers for turbine blade cooling [4]. Control of the jet in crossflow is typically accomplished through temporal excitation of the jet fluid using flowrate modulation or acoustic forcing [5], [6], [7], [8]. The excitation is periodic, usually with the goal of forming either sinusoidal or pulse-like jet velocity profiles.

In practice, feedback control is required to shape the jet velocity since open-loop methods show significant amount of ringing and asymmetry compared to the reference waveforms [7], [8]. The error is a result of both disturbances and unmodeled system dynamics. The field of repetitive control, which addresses asymptotic disturbance rejection and reference tracking of periodic signals, provides a framework for pulsed jet reference tracking. Systems based on repetitive control commonly use a time delay in the feedback loop to place an infinite number of poles on the imaginary axis at

This work is sponsored by the National Science Foundation under grant no. CBET-0755104

C. Hendrickson and R.T. M'Closkey are with the Department of Mechanical and Aerospace Engineering, University of California, Los Angeles, Los Angeles, CA, 90095 USA rtmeseas. ucla.edu the fundamental frequency and harmonics of the periodic disturbance or reference [9], [10]. In practice, the plant has a finite bandwidth, therefore, only a finite number of internal models are required for asymptotic tracking. In this case, modulated-demodulated control can be used as an alternative to time delay repetitive control [11], [12]. Modulated-demodulated control, also referred to as adaptive feedforward control or adaptive feedforward cancellation [13], demodulates the spectrum of "high" frequency oscillations to baseband signals, operates at baseband, and then modulates the baseband spectrum back to high frequency.

In the pulsed jet experiment, the plant represents the dynamic system from the actuator command to the hotwire anemometer measurement at the jet exit. At high forcing amplitudes (more than $10 \%$ of mean jet velocity -see [14]) the jet velocity, if following a periodic reference, exhibits nontrivial coupling between harmonics that can destabilize the closed-loop system with a controller designed for more moderate forcing amplitudes. We show that identification of the plant dynamics in the baseband coordinates provides key insight into the nonlinearity that couples adjacent frequency "channels" and provides a convenient means for compensating the coupling. In fact, the nonlinear coupling can be modeled in the baseband coordinates as a MIMO constant gain. This control strategy achieves asymptotic tracking of the jet velocity within the bandwidth of control.

\section{Pulsed Jet Experiment}

\section{A. Actuation System}

A schematic of the experimental pulsed jet injection apparatus is shown in Fig. 1. Compressed air, regulated to maintain a constant mean jet velocity of $8 \mathrm{~ms}^{-1}$, flows into a plenum then through a smoothly contracted nozzle into quiescent surroundings. The jet velocity is perturbed about its $8 \mathrm{~ms}^{-1}$ mean value by a lightweight piston positioned at the bottom of the plenum. The piston is driven inline with the jet by a modal shaker. The shaker voice coil current is proportional to the shaker amplifier input signal. The amplifier input signal is the plant input. The jet velocity is measured using a hotwire anemometer (Dantec 54T30) placed in the center of the jet at the nozzle exit. Additionally, the apparatus is equipped with a microphone (PCB Piezotronics 378C01) located at the top of the plenum. The controllers are implemented in Matlab's XPC Target application with a $25 \mathrm{kHz}$ sampling rate. Two, 8-pole lowpass Chebyshev filters with $10 \mathrm{kHz}$ corner frequencies filter the microphone and hotwire signals prior to sampling. We 
demonstrate our control technique without crossflow, however, the entire system can be placed beneath a wind tunnel with the nozzle exit flush with the test section floor if a crossflow is desired.

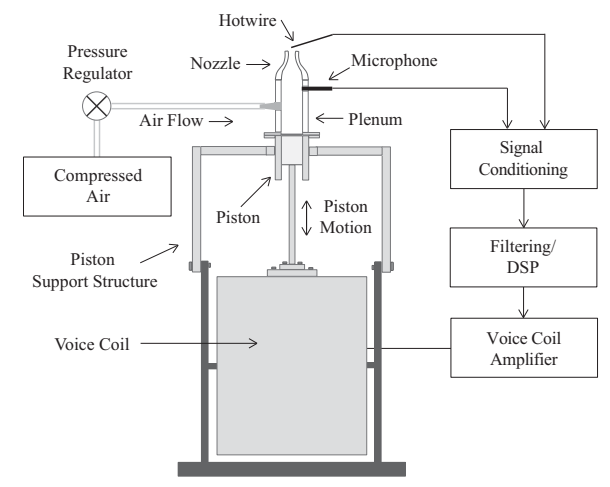

Fig. 1. Pulsed jet injection experimental setup using a piston to actively control the temporal velocity waveform of a jet at the nozzle exit.

\section{B. Motivation: nonlinear coupling between harmonics}

Linear models of the pulsed jet can be developed from data generated with low amplitude test inputs. Controllers developed using the models are then adequate for low amplitude reference tracking [14], [15]. At RMS amplitudes exceeding $0.5 \mathrm{~ms}^{-1} \mathrm{RMS}$, though, the nonlinear response of the velocity measurement (the regulated variable) can destabilize the closed-loop system with these controllers. The desired velocity perturbation is periodic so the nonlinear response can be studied from the point of view of coupling among harmonics in the periodic waveform. The magnitude and character of the harmonic coupling is dependent upon the desired velocity reference and the mean jet velocity. In general, the velocity output at given harmonic is a function of the input energy at all harmonics. For example, the velocity "frequency response", shown in Fig. 2, is measured using band-limited white noise inputs with amplitudes set to perturb the jet velocity by $0.15 \mathrm{~ms}^{-1}$ RMS (solid line) and $0.80 \mathrm{~ms}^{-1}$ RMS (dashed line). The frequency response is determined from averaging the cross-spectra of the inputoutput data. It is evident that the frequency response derived from the case with harder forcing deviates from the nominal, or low amplitude, frequency response. Additionally, the jet velocity coherence, shown in Fig. 3, decreases with the larger amplitude forcing compared to the nominal case. The coherence is reduced because a greater portion of the velocity output is determined by nonlinear dynamics at the larger forcing amplitude. In contrast, the frequency responses with the pressure measurement are nearly identical for both test amplitudes and, furthermore, Fig. 3 shows the pressure coherence increases with harder forcing (the expected response of a linear plant with an additive fixed disturbance/noise spectrum).

The harmonic coupling in the jet velocity is clearly illustrated by the spectra in Fig. 4a produced in response to dual tone forcing. Input tones at $1800 \mathrm{~Hz}$ and $1900 \mathrm{~Hz}$ creating a
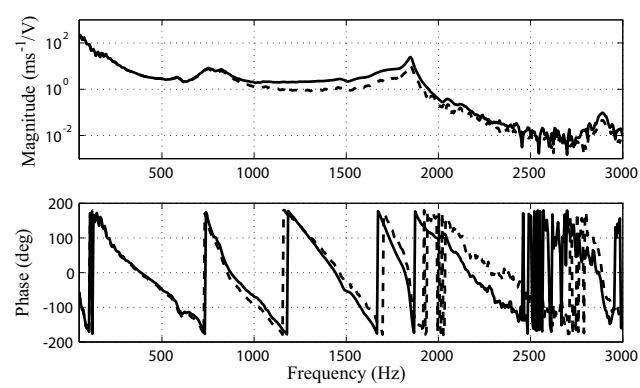

Fig. 2. Velocity frequency responses obtained with a white noise identification input which perturbs the jet velocity by $V_{r m s}=0.15 \mathrm{~ms}^{-1}$ (solid) and $V_{r m s}=0.80 \mathrm{~ms}^{-1}$ (dashed). The velocity frequency response is dependent on the forcing condition whereas the pressure measurement is independent of the forcing condition.

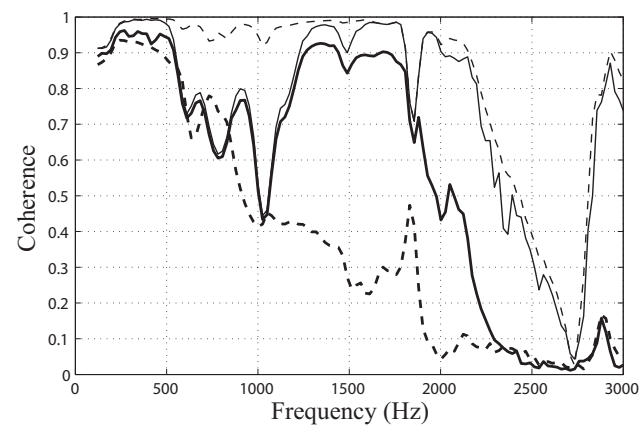

Fig. 3. Coherence of the empirical velocity frequency response data in Figures 2 and the empirical microphone frequency response (not shown). The $0.80 \mathrm{~ms}^{-1}$ RMS microphone coherence (thin dahsed line) increases from the $0.15 \mathrm{~ms}^{-1}$ RMS microphone coherence (thin solid line) due to improved signal-to-noise ratio while the the $0.80 \mathrm{~ms}^{-1}$ RMS velocity coherence (thick dashed line) mostly decreases from the $0.15 \mathrm{~ms}^{-1}$ RMS velocity coherence (thick solid line) due to nonlinear distortion.

$0.50 \mathrm{~ms}^{-1}$ RMS perturbation produce strong super- and subharmonics. On the other hand, Fig. 4b shows the microphone spectrum in response to the same dual tone input as above - the microphone measurement is dominated by tones at $1800 \mathrm{~Hz}$ and $1900 \mathrm{~Hz}$.

\section{IDENTIFICATION OF HARMONIC COUPLING}

The frequency responses in Fig. 2 show that the magnitudes roll off after a plenum mode near $1.8 \mathrm{kHz}$. This limits the actuation bandwidth to approximately $2.0 \mathrm{kHz}$. As such, we specify the periodic reference to be truncated at or below $2 \mathrm{kHz}$ to avoid saturation of the actuator amplifier. Throughout this paper the reference waveforms are periodic square pulses with a $100 \mathrm{~Hz}$ fundamental frequency so the modulation-demodulation is centered in narrow bands around the $N=20$ harmonics within the $2 \mathrm{kHz}$ actuation bandwidth. Although the disturbance spectrum will only be attenuated in neighborhood of each harmonic, the primary reason for using feedback to shape the jet velocity is the uncertainty associated with the plant dynamics. Thus, feedback is used to force the jet velocity to asymptotically track the periodic reference within the actuator bandwidth, even in the presence of the significant coupling noted in Fig. 4. The physical mechanism causing the nonlinear harmonic coupling is not 

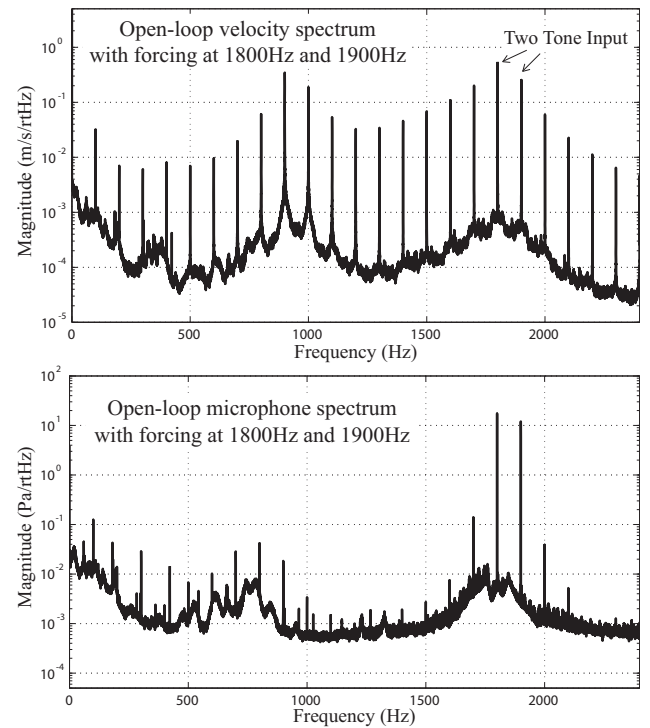

Fig. 4. (Top) Velocity response to dual tone forcing at $1800 \mathrm{~Hz}$ and $1900 \mathrm{~Hz}$. The harmonic coupling appears at harmonics of $100 \mathrm{~Hz}$, the difference between $1800 \mathrm{~Hz}$ and $1900 \mathrm{~Hz}$. (Bottom) Pressure response to dual tone forcing at $1800 \mathrm{~Hz}$ and $1900 \mathrm{~Hz}$. The output is dominated by the linear response at $1800 \mathrm{~Hz}$ and $1900 \mathrm{~Hz}$.

well understood, however, an empirical model can be built that is quite suitable for analysis and controller synthesis. We identify a model which characterizes the harmonic coupling in the neighborhood of a particular periodic jet velocity operating point which is close to the desired periodic reference.

At a given operating point, though, the main challenge in modeling the system is quantifying the nonlinear coupling that occurs between the $N$ frequency "channels" of the hotwire signal. The identification is facilitated by shifting the spectrum of the plant's input and output in the neighborhood of each harmonic to "baseband" via modulation and demodulation. The architecture is shown in Fig. 5, in which both plants, $P_{m i c}(s)$ and $P_{v e l}$, are transformed into "demodulated" plants represented as $2 N \times 2 N$ MIMO systems. Since the plant based on the microphone measurement is wellmodeled as a linear system, we use the transfer function notion with argument $s$, whereas, the plant with hotwire measurement is genuinely nonlinear and must be analyzed as such. The demodulated plants with microphone measurement and hotwire measurement are denoted $\tilde{P}_{m i c}(s)$ and $\tilde{P}_{v e l}$, respectively. Each of the $N$ frequency channels possesses an in-phase and quadrature input, denoted $u_{n}^{i}(t)$ and $u_{n}^{q}(t)$, and an in-phase and quadrature output, denoted $y_{n}^{i}(t)$ and $y_{n}^{q}(t)$. The low-pass filter, denoted by the $H_{l p}$ block, restricts the demodulated plant bandwidth. The low-pass filter corner frequency, denoted $\omega_{c}$, is chosen to be $\omega_{c}<\frac{\omega_{f}}{2}$ since this prevents any direct overlap of, and interaction between, adjacent channels.

The demodulated microphone plant, $\tilde{P}_{\text {mic }}(s)$, is $2 \times 2$ block diagonal and independent of the operating point since $P_{\text {mic }}$ is essentially linear. In contrast, the demodulated plant, $\tilde{P}_{v e l}$, is, in general, full and dependent upon the system operating point. Interestingly, though, in a neighborhood of an operat-

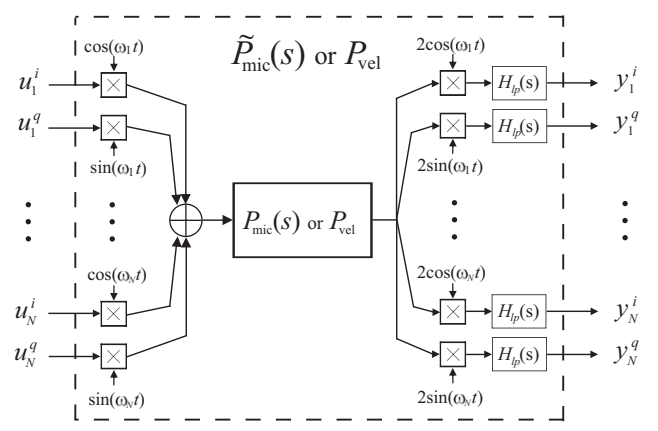

Fig. 5. Diagram of the "demodulated" plant in which narrow band control in a neighborhood of the $N$ harmonics yields a $2 N$ input- $2 N$ output system of low bandwidth dynamic systems. If the plant is linear then the demodulated plant is $2 \times 2$ block diagonal.

ing point $\tilde{P}_{v e l}$ can be modeled as an affine function of the input. The transformation to baseband coordinates allows us to characterize the nonlinear harmonic coupling phenomenon in a linear framework which simplifies identification and control.

\section{A. Inner loop with microphone feedback}

Although it is possible to use the jet velocity exclusively for feedback, the large variation in plant gain (two orders of magnitude over the usable bandwidth vis à vis Fig. 2) implies that $\tilde{P}_{v e l}$ will have a large condition number since the $2 \times 2$ block diagonal terms will follow the trend in the estimated frequency response. The large condition number makes the inversion-based compensator described in Section IV susceptible to errors in the identified models. This can be overcome by using an inner microphone loop which equalizes the gain from the microphone references to the jet velocity. The block diagram is shown in Fig. 6 where $\tilde{r}_{m i c}$ represents the vector of reference signals for the demodulated microphone plant and $\tilde{u}$ represents the output of the microphone controller $\tilde{C}_{m i c}$. The transfer function from $\tilde{r}_{m i c}$ to $\tilde{u}$ approximately inverts $\tilde{P}_{m i c}$ in a neighborhood of the harmonics. When this inverse is cascaded with $\tilde{P}_{v e l}$, the relationship between $\tilde{y}_{v e l}$ and $\tilde{r}_{m i c}$ does not suffer from the large gain variations in the block diagonal terms.
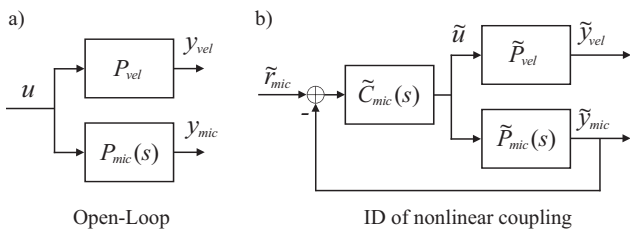

Fig. 6. a) Open-loop plant. b) Block diagram for identification of harmonic coupling. The inner control loop establishes an operating point which drives $\mathrm{y}_{v e l, d}$ close to the desired waveform. The microphone and velocity demodulated plants are denoted $\tilde{P}_{m i c}(s)$ and $\tilde{P}_{v e l}$, respectively.

The inner loop is closed around $\tilde{P}_{\text {mic }}(s)$ using the modulated-demodulated control method described in [14]. The inner loop controller is $2 \times 2$ block diagonal like $\tilde{P}_{m i c}(s)$. Fig. 7 shows the $n$th channel of $\tilde{C}_{m i c}(s)$. The integrators provide zero steady state error in tracking each reference 
and the constant gains $R_{n}$ and $I_{n}$ invert the phase of $\tilde{P}_{m i c}$ at frequency $\omega_{n}$, i.e. $R_{n}+j I_{n}=\frac{P_{\text {mic }}\left(j \omega_{n}\right)}{\left\|P_{\text {mic }}\left(j \omega_{n}\right)\right\|}$. Detailed analysis of the dynamics of the inner loop from both the measurement and the baseband perspectives are given in [14], [15] so details are not provided due to space constraints, however, it should be noted that the inner loop can be represented as a set of $N$ uncoupled linear time-invariant subsystems, each with loop transfer function

$$
\begin{array}{r}
L_{m i c, n}(s)=g_{m i c} P_{m i c}(s)\left[\frac{H_{l p}\left(s-j \omega_{n}\right)\left(R_{n}-j I_{n}\right)}{s-j \omega_{n}}\right. \\
\left.+\frac{H_{l p}\left(s+j \omega_{n}\right)\left(R_{n}+j I_{n}\right)}{s+j \omega_{n}}\right]
\end{array}
$$

where $g_{m i c}$ is an adjustable gain that controls the time constant of the $n$th channel.

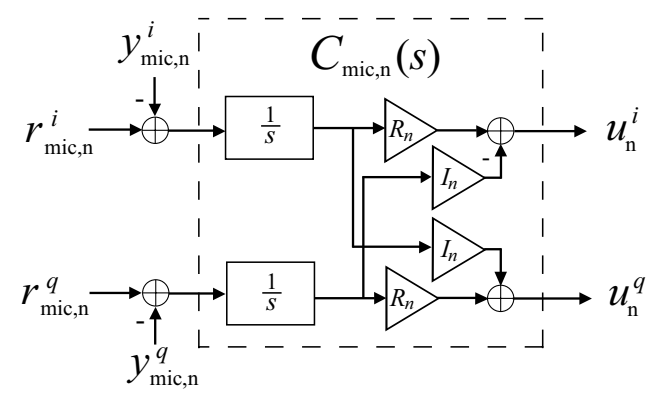

Fig. 7. Channel $n$ microphone Controller

The coupling between channels in $\tilde{P}_{v e l}$ is a function of the operating point so it is necessary to specify a constant pressure reference vector $\bar{r}_{\text {mic }}$ such that the elements of $\tilde{y}_{v e l}$ are close to the values associated with the Fourier series of the desired periodic jet velocity waveform. The coupling is identified about the operating condition by adding a time varying perturbation to the constant reference. In other words, the reference input in Fig. 6 is specified to be $\tilde{r}_{m i c}=$ $\bar{r}_{m i c}+\delta_{m i c}$, where $\delta_{m i c}$ is the perturbation employed for identification in a neighborhood of the operating condition established by $\bar{r}_{m i c}$.

If we treat $P_{v e l}$ as a linear system, then the in-phase and quadrature components of $\bar{r}_{m i c}$ associated with the $n$th frequency are chosen to be

$$
\bar{r}_{m i c, n}^{i}+j \bar{r}_{m i c, n}^{q}=P_{m i c}\left(j \omega_{n}\right) \bar{P}_{v e l}^{-1}\left(j \omega_{n}\right) r_{v e l, n}
$$

where $r_{v e l, n}$ is the Fourier coefficient of the desired jet velocity signal at frequency $\omega_{n}$, and where $\bar{P}_{v e l}\left(j \omega_{n}\right)$ is chosen from the "linear" jet response in Fig 2. This method does not compensate for the nonlinear dynamics of $P_{v e l}$ and, therefore, $y_{v e l}$ does not actually track the desired periodic jet velocity waveform. For example, Fig. 8 compares the measured velocity waveform taken with the microphone reference selected in this manner (solid line) to its $20 \%$ duty cycle, $V_{r m s}=0.9 \mathrm{~ms}^{-1}$ desired reference (dashed line). Although the measured waveform does not track the velocity reference, it is close enough to identify a model of the harmonic coupling which is then employed to design a stabilizing "outer" loop using feedback from the hotwire.

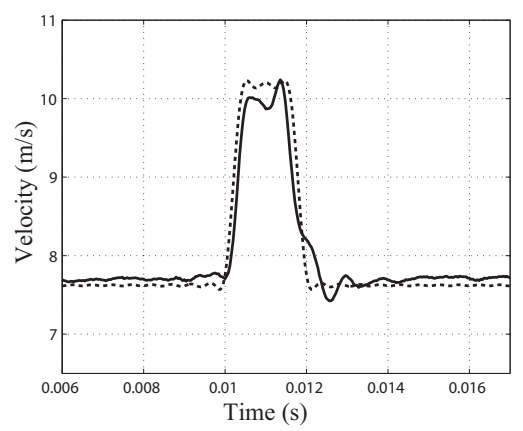

Fig. 8. Jet velocity for a $V_{r m s}=0.9 m s^{-1}, 20 \%$ duty cycle velocity reference using only microphone feedback with the microphone reference chosen using Eq. (2). The measured waveform is shown in the solid line and the velocity reference is shown in the dashed line.

\section{B. Harmonic coupling identification results}

With the inner loop closed, the dynamics from $\tilde{r}_{m i c}$ to the demodulated jet velocity $\tilde{y}_{v e l}$ can be identified in a neighborhood of the operating point by applying independent, low bandwidth, zero-mean random perturbations to the inphase components of $\tilde{r}_{m i c}$. In response to the microphone reference, $\tilde{r}_{m i c}=\bar{r}_{m i c}+\delta_{m i c}$, the demodulated velocity components are recorded, $\tilde{y}_{v e l}=\bar{y}_{v e l}+\delta_{v e l}$, where $\delta_{v e l}$ is the time-varying component of $\tilde{y}_{v e l}$. For example, Fig. 9 presents selected empirical frequency response estimates from $\delta_{m i c}$ to $\delta_{v e l}$ at two operating points: the unforced operating point and the $20 \%$ duty cycle, $V_{r m s}=0.9 \mathrm{~ms}^{-1}$ operating point. In particular, the figures show the identified frequency response magnitudes when a test perturbation is applied to the 15th and 16th input channels and the subsequent response of the 15th and 16th demodulated jet velocity output channels is measured. For the case where the reference Fourier coefficients are zero, there is very little cross channel coupling. In marked contrast to this result, if non-zero reference coefficients are specified yielding an operating condition in which $V_{r m s}=0.9 \mathrm{~ms}^{-1}$, then not only have the "diagonal" transfer functions changed, but the off-diagonal gains are now non-zero and in some cases as large as the diagonal gains. This cross-channel coupling is a nonlinear phenomenon because it shows that a sinusoidal perturbation at one frequency produces responses across a range of frequencies.

An efficient method is required for determining this coupling due to the high channel count in the input and output variables $\tilde{r}_{m i c}$ and $\tilde{y}_{v e l}$. The measurements in Fig. 9 suggest that if the bandwidth of the compensator using $\tilde{y}_{v e l}$ for feedback is sufficiently low, then only the DC gains of the elements are required. This is in fact how we proceed so the following model is used to relate $\delta_{m i c}$ and $\delta_{v e l}$

$$
\delta_{\text {vel }}=K \delta_{m i c}
$$

where $\mathrm{K}$ is a constant $2 N \times 2 N$ real matrix. For example, the outputs in channel $n$ are related to the inputs in channel $p$ by

$$
\left[\begin{array}{l}
\delta_{v e l, n}^{i} \\
\delta_{v e l, n}^{q}
\end{array}\right]=\left[\begin{array}{cc}
\alpha_{n, p}^{i} & -\alpha_{n, p}^{q} \\
\alpha_{n, p}^{q} & \alpha_{n, p}^{i}
\end{array}\right]\left[\begin{array}{c}
\delta_{m i c, p}^{i} \\
\delta_{m i c, p}^{q}
\end{array}\right]
$$



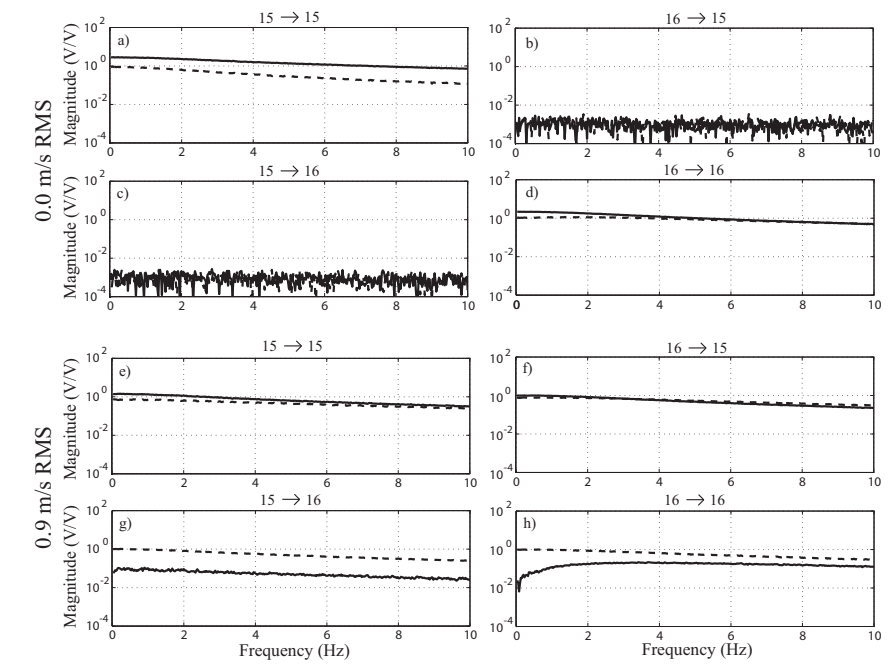

Fig. 9. System identification magnitude shown for the $n=15$ and $n=16$ input and output channel. The system is identified at the unforced operating point (a-d) and the $20 \%$ duty cycle, $V_{r m s}=0.9 \mathrm{~ms}^{-1}$ operating point (e-h). The transfer functions to the in-phase outputs are shown in the solid lines and to the quadrature outputs in the dashed lines. Little cross channel coupling occurs at $V_{r m s}=0 \mathrm{~ms}^{-1}$ in contrast to the large cross channel coupling at $V_{r m s}=0.9 \mathrm{~ms}^{-1}$.

where coefficients $\alpha_{n, p}^{i}$ and $\alpha_{n, p}^{q}$ are constant and real. The matrix $K$ is composed of the identified $\alpha$ 's assembled in $2 \times 2$ blocks as shown in (4) where the $\alpha_{n, p}^{i}$ coefficients appear in the $(2 n-1,2 p-1)$ and $(2 n, 2 p)$ elements of $K$. Because of the structure of $K$, only $N$ identification inputs are needed to identify the $\alpha$ 's and so all identification inputs are simultaneously applied to the $\delta_{m i c, k}^{i}, k=1, \ldots, N$, input variables with the bandwidth of each perturbation constrained to $0.2 \mathrm{~Hz}$ in order emphasize the low frequency power. In other words,

$$
\begin{aligned}
& \delta_{v e l, n}^{i}(t)=\alpha_{1, n}^{i} \delta_{m i c, 1}^{i}(t)+\cdots+\alpha_{N, n}^{i} \delta_{m i c, N}^{i}(t) \\
& \delta_{v e l, n}^{q}(t)=\alpha_{1, n}^{q} \delta_{m i c, 1}^{i}(t)+\cdots+\alpha_{N, n}^{q} \delta_{m i c, N}^{i}(t),
\end{aligned}
$$

where $n=1, \ldots, N$. The $\alpha$ 's are chosen to minimize the RMS of the residuals associated with each expression in (5) using a given IO data set. Fig. 10 shows an example comparing the predicted $\delta_{v e l}$ to the measured value for the in-phase $n=15$ output.

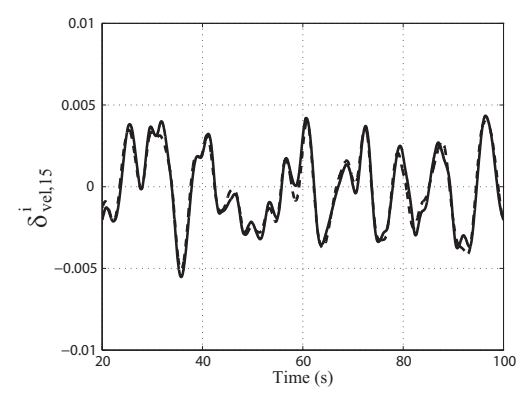

Fig. 10. Response of demodulated hotwire output $y_{v e l, 15}^{i}$ to identification at the $20 \%$ duty cycle, $V_{r m s}=0.9 \mathrm{~ms}^{-1}$ operating point. The measured signal (solid line) is compared to the predicted output using (5) (dashed line). The error is small in comparison to the measured perturbation.

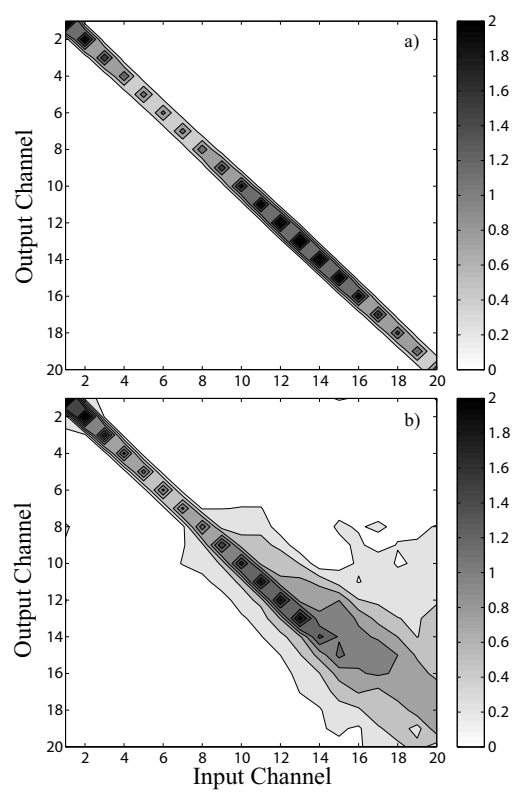

Fig. 11. Graphical representation of $K$ at a) the unforced operating point and b) at a $20 \%$ duty cycle, $V_{r m s}=0.9 \mathrm{~ms}^{-1}$ operating point

There is an graphical means to reveal the coupling between frequency channels by computing the maximum singular value of each $2 \times 2$ sub-block (4) of $K$ and replacing the subblock with a grayscale shade that denotes the value of the norm. Fig. 11 illustrates two such maps, one at the unforced operating point (a), and the other at the $20 \%$ duty cycle, $V_{r m s}=0.9 \mathrm{~ms}^{-1}$ operating point (b). At the unforced operating point, $K$ is essentially block diagonal which indicates little-to-no harmonic coupling. This is representative of a linear response in which an input perturbation at one frequency only effects that same frequency in the output. In contrast, at the $V_{r m s}=0.9 \mathrm{~ms}^{-1}$ operating point, the identified $K$ shows a strong level of harmonic coupling among frequencies above the $n=8$ channel.

\section{COMPEnSATION OF HARMONiC COUPLinG}

At a particular operating point asymptotic tracking of the desired periodic reference is implemented as shown in the block diagram of Fig. 12. Feedback of the demodulated hotwire signals tracks the Fourier series of the periodic reference, arranged in the $2 N$ vector $\tilde{r}_{v e l}$ as

$$
\tilde{r}_{v e l}=\left[\begin{array}{lllll}
r_{v e l, 1}^{i} & r_{v e l, 1}^{q} & \ldots & r_{v e l, N}^{i} & r_{v e l, N}^{q}
\end{array}\right]^{T} .
$$

The controller, denoted $C_{v e l}(s)$, is $2 N \times 2 N$ and, in general, full. The system in the dashed box, identified using the procedure outlined in the previous section, is modeled as a $2 N \times 2 N$ static map for the purpose of controller synthesis.

We use an integral based controller to compensate the identified harmonic coupling and asymptotically track a periodic reference. A model inverse controller with

$$
C_{v e l}(s)=g_{v e l} K^{-1} \frac{1}{s} I
$$




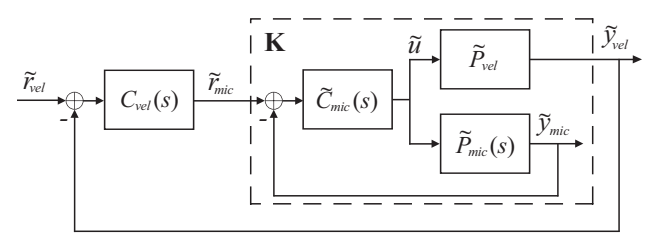

Fig. 12. Diagram for control of harmonic coupling.

where $g_{v e l}$ is a positive gain used to control the outer loop convergence rate, is a straightforward approach for velocity regulation in the neighborhood of a single operating point. In general, $K$ must be well conditioned to use (7). The microphone feedback loop is needed to decrease the condition number of the identified static map by "equalizing" the identified system's frequency response magnitude from the SISO perspective. The condition number of $K$ is $\kappa=2.7$ at the unforced operating point and $\kappa=8.3$ at $V_{r m s}=$ $0.9 m s^{-1}$.

The modeled system's closed-loop eigenvalues simplify to the eigenvalues of $-g_{v e l} I$ at the operating point, indicating the closed-loop system has equal convergence at all frequencies of control with a closed loop time constant $\tau_{v e l}=$ $1 / g_{\text {vel }}$. In practice, the harmonic coupling with the system converged to the reference slightly differs from the harmonic coupling at the operating point. The converged closed-loop system dynamics, therefore, can only be approximated by the static model.

Experimental results for asymptotic tracking of a $20 \%$ duty cycle, $V_{r m s}=0.9 \mathrm{~ms}^{-1}$ RMS square wave are presented in Fig. 13. This figure demonstrates that the empirical waveform with microphone and hotwire feedback (solid line) closely matches the $20 \%$ duty cycle, $V_{r m s}=0.9 \mathrm{~ms}^{-1}$ velocity reference (dashed line), the same reference as in Fig. 8 for microphone-only feedback. A hotwire feedback controller is synthesized using (7) and the static map visualized in Fig. 11b. The outer feedback loop asymptotically converges the hotwire Fourier coefficients to the reference waveform coefficients appearing in (6). There are small periodic deviations evident in the time series, however, which are the result of harmonics excited beyond $2.0 \mathrm{kHz}$. As these harmonics lie beyond the bandwidth of the actuation system, they are uncontrollable.

\section{CONCLUSION}

In this paper we presented a strategy to identify and control nonlinear coupling for periodic reference tracking in a pulsed jet injection experimental study. A data-driven approach is needed, whereby the nonlinear coupling is identified in the neighborhood of a particular forcing condition. A static linear model accurately characterizes the nonlinear coupling in the neighborhood of the operating point after a change of coordinates to baseband. In this reference frame, a stabilizing feedback can be synthesized from a static map that captures the coupling between frequency channels and is shown to asymptotically track a periodic reference at its first 20 harmonics at an operating condition with significant

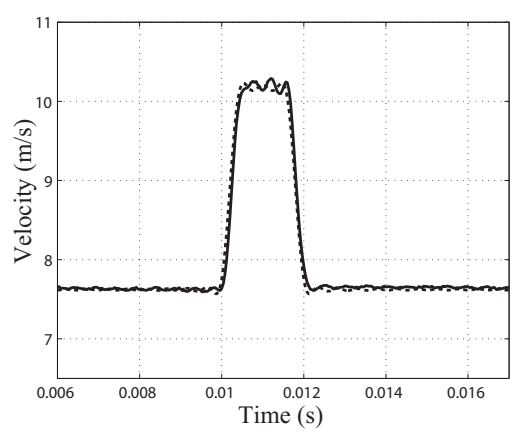

Fig. 13. Jet velocity with a $V_{r m s}=0.9 \mathrm{~ms}^{-1}, 20 \%$ duty cycle velocity reference with the outer loop closed around the hotwire measurement. The Fourier coefficients of the jet velocity asymptotically converge to the reference waveform coefficients. The dashed line is the reference waveform.

nonlinear harmonic coupling.

\section{ACKNOWLEDGMENT}

The authors gratefully acknowledge the the advice of our colleague Ann Karagozian and the financial support of the National Science Foundation.

\section{REFERENCES}

[1] R. J. Margason, "Fifty years of jet in crossflow research," $A G A R D-C P$, vol. 534, pp. 1-141, 1993.

[2] A. R. Karagozian, "Transverse jets and their control," Progress in Energy and Combustion Science, vol. 36, no. 5, pp. 531-553, 2010.

[3] P. J. Vermeulen, P. Grabinski, and V. Ramesh, "Mixing of an acoustically excited air jet with a confined hot crossflow," Journal of Engineering for Gas Turbines and Power, vol. 114, pp. 46-54, 1992.

[4] J. P. Bons, R. Sondergaard, and R. B. Rivir, "Journal of turbomachinery," Progress in Energy and Combustion Science, vol. 124, no. 1, pp. 77-85, 2002.

[5] H. Johari, M. Pacheco-Tougas, and J. C. Hermanson, "Penetration and mixing of fully modulated turbulent jets in crossflow," AIAA Journal, vol. 37, no. 7, pp. 842-850, 1999.

[6] R. T. M'Closkey, J. King, L. Cortelezzi, and A. R. Karagozian, "The actively controlled jet in crossflow," Journal of Fluid Mechanics, vol. 452, pp. 325-335, 2002.

[7] S. Shapiro, J. King, R. T. M'Closkey, and A. R. Karagozian, "Optimization of controlled jets in crossflow," AIAA Journal, vol. 44, pp. 1292-1298, 2006.

[8] J. Davitian, C. Hendrickson, D. Getsinger, R. M'Closkey, and A. Karagozian, "Strategic control of transverse jet shear layer instabilities," AIAA Journal, vol. 48, no. 9, pp. 2145-2156, 2010.

[9] T. C. Tsao and M. Tomizuka, "Robust adaptive and repetitive digital tracking control and application to a hydraulic servo for noncircular machining," Journal of Dynamical Systems, Measurement, and Control, vol. 116, pp. 24-32, 1994.

[10] K. K. Chew and M. Tomizuka, "Digital control of repetitive errors in disk drive systems," IEEE Control Systems Magazine, vol. 10, pp. 16-20, 1990.

[11] K. Lau, G. C. Goodwin, and R. T. M'Closkey, "Properties of modulated and demodulated systems with implications to feedback limitations," Automatica, vol. 41, pp. 2123-2129, 2005.

[12] K. Lau, D. E. Quevedo, B. J. G. Vautier, G. C. Goodwin, and S. O. R. Moheimani, "Design of modultated and demodulated controllers for flexible structures," Control Engineering Practice, vol. 15, pp. 377388, 2005.

[13] M. F. Byl, S. J. Ludwick, and D. L. Trumper, "A loop shaping perspective for tuning controllers with adaptive feedforward cancellation," Precision Engineering, vol. 29, pp. 27-40, 2005.

[14] C. Hendrickson and R. T. M'Closkey, "Phase compensation strategies for modulated-demodulated control with application to pulsed jet injection," J. Dyn. Sys., Meas., Control, vol. 134, no. 1, 2012.

[15] — , "Dynamic phase compensation in modulated-demodulated control for pulsed jet injection," Proceedings of the American Control Conference, pp. 3053-3058, 2011. 